\title{
Penile Prosthesis Surgery in Italy: Personal Experiences, Compli- cations and Considerations after 552 Cases
}

\author{
Diego Pozza ${ }^{1 *}$, Mariangela Pozza ${ }^{1}$ and Carlotta Pozza ${ }^{2}$ \\ ${ }^{1}$ Studio di Andrologia e di Chirurgia Andrologica, Rome, Italy \\ ${ }^{2}$ Department of Experimental Medicine, Sapienza University, Rome, Italy
}

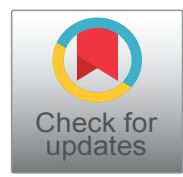

*Corresponding author: Diego Pozza, MD, Studio di Andrologia e di Chirurgia Andrologica, Rome, Italy

\begin{abstract}
The Implant of Penile Prostheses (Silicone, Malleable, Inflatable) represents an efficient solution for patients affected by Erectile Dysfunction (ED) and penetrating incapacity who do not respond to the other treatment modalities (PDE5i, PGE1, Vacuum Systems). Patients with penile prostheses regain the spontaneity of intercourses without having to assume drugs, to introduce mechanically medical substances or to activate complex mechanisms. Just like the whole field of prosthetic surgery, introducing into the organism a "foreign body" can lead to complications connected to the patient, the inserted matter, the inserting surgical procedure and the after-care. A "relevant" complication - which almost always leads to the removal of the implant and thus to the failure of the surgeon's treatment and to the extreme disappointment of the patient's expectations -can often be considered as result of an initially "small" complication, which could be faced and solved (with drugs or a minor surgery) without having to resort to removal. The authors present a variety of medical and surgical procedures, derived from their over 34-year experience (1984-2018) in Penile Prosthesis Surgery (PPS), which may be implemented to avoid implant failure. Plenty of other complications are possible and have been registered in Medical Literature. They are often caused by reactions of the penile tissues, by vascular or hemorrhagic phenomena, by modifications in the penile or glans sensibility.
\end{abstract}

\section{Introduction}

Penile Prosthetic Surgery (PPS) should take care of the specific genital structures avoiding of interfering with ejaculation and micturition, obtaining a good aesthetic result with a conclusive result, not particularly invasive, both aesthetically and functionally acceptable [1-6]. Infection is considered the worst complication in prosthetic surgery [7], difficult to cure with usual antibiotic therapy [8]. The use of new IPP such as AMS 700 with InhibiZone, (antibiotic surface treatment), or Coloplast hydrophilic coating (which absorbs and releases antibiotic) has led to a significant reduction, but not eradication, in the rate of infections $[9,10]$. The mechanical breakdown of Inflatable Penile Prostheses (IPP) [11], which can cause them to malfunction is another possible complication. In such a case, the implant must be removed and replaced with a similar system. Leading Manufacturers guarantee their replacement in the event of malfunction. However, the emotional effects and the financial cost of a new surgical procedure, often not covered by Health Insurance, are negative factors for patients [12]. In some cases, the surgical maneuvers performed to create a space for the reservoir in the peritoneal cavity o in the peri vesical space could provoke intestinal herniation, torsion or perforation, hematoma of the abdominal wall and bladder perforation $[11,13]$. AMS and Coloplast have recently introduced new reservoirs which are easier to insert and more adaptable, (Conceal and Cloverleaf) [13-16]. A frequent complication is determined by the incorrect sizing of the cavernous spaces, which can lead to the insertion of inadequate cylinders which could erode or perforate the corpora cavernosa or affect the symmetry of the penis (SST effect) [17]. In general, many complications encountered with penile surgery are highly dependent on the surgeon's experience [18]. Diabetes, Urinary Tract Infections, Blood Coagulation disorders, immunodepression often present in this kind of patients such as duration of hospitalization,

Citation: Pozza D, Pozza M, Pozza C (2018) Penile Prosthesis Surgery in Italy: Personal Experiences, Complications and Considerations after 552 Cases. Int Arch Urol Complic 4:050. doi.org/10.23937/2469$5742 / 1510050$

Accepted: December 17, 2018: Published: December 19, 2018

Copyright: (C) 2018 Pozza D, et al. This is an open-access article distributed under the terms of the Creative Commons Attribution License, which permits unrestricted use, distribution, and reproduction in any medium, provided the original author and source are credited. 
inappropriate surgical technique and prolonged surgery time should all be assessed. A psychological pre-op assessment should be considered important, to understand if the patient is aware not only of the benefits of a penile implant but also of its impact on his personal and relational life [12].

\section{Material and Methods - Case Reports}

552 penile prostheses have been implanted from 1984 to 2018 in the Studio di Andrologia e di Chirurgia Andrologica of Rome. All the patients had been visited, operated and followed by the same surgeon (DP) [19]. It is very difficult to have the economic support of the Italian National Health Service to cover the expenses of these procedures. Most of our patients had to pay all costs. Few patients had private Health Insurances covering in part the medical expenses. Sometimes our operating decisions were influenced by economic considerations of the patient. They certainly influenced the choice of the prosthetic implant. We implanted 193 semi-rigid silicone (Eurogest, Implantal, Small Carrion, Subrini, SSDA-GIS), 191 malleable (Jonas, Omniphase, Duraphase, Vedise, AMS600, Mentor Acuform, Coloplast Genesis). We implanted the most expensive IPP only in 168 cases (Mentor Mark II, Alpha I, Titan OTR, AMS Hydroflex, Dynaflex, Ambicor, 700 Ultrex, 700CX, 700LGX). We report the complications observed in our surgical experience.

\section{Infectious Complications}

The correct antibiotic therapy able to eliminate or diminish the incidence of infections of penile prostheses continues to be researched and debated [20,21]. All patients were recommended to clean their genitals with iodopovidone fluid twice a day for the 3 days before surgery. Skin shaving was performed the morning of surgery. Since 1991 (114 patients) we utilized an antibiotic treatment with Gentamicin and second generation Cephalosporins, starting 1 day before operation till 7 days post op. After having recorded several untreatable infections in $2 / 96(2.08 \%)$ patients with not inflatable and in 3/18 (16.2\%) with inflatable Prostheses, in 1991 we changed the antibiotic protocol. Since 1992 (438 patients) we utilize Ciprofloxacin $1000 \mathrm{mg} /$ day since 3 days pre-op till 7 days post-op; Ceftriaxone 1000 mg/day from operation till 7 days post-op; Gentamicin $160 \mathrm{mg} /$ day since operation till 2 days post-op.

With the new antibiotic protocol, we recorded untreatable infections in 4/288(1.4\%) with Not Inflatable Prostheses and in 13/150 (8.0\%) with Inflatable ones. The rate of untreatable infections continues to be considered the worst complication of penile PPS. In our experience in some peculiar cases an infection could be afforded with medical therapy [19].

-In 2004, a 58 years old patient with vascular ED, received an AMS700 Ultrex IPP. Usual pre- and postoperative antibiotic therapy. After 2 weeks, before the implant had been used, the patient complained of pain at the base of the penis, septic fever and a yellowish boil at the base of the penis on the right, with a yellowish creamy purulent secretion (Figure 1). If we inflated the cylinders the volume of pus increased. Due to the purulent infection, it was suggested to the patient that the implant should be removed. The patient, mainly for economic reasons, refused categorically. We began with a local injection of a solution of iodopovidone and Gentamicin, 2-3 times a day, with deflated cylinders, followed by the partial inflation of prosthetic cylinders in order to wash and evacuate the infected cavernosal fluid. We repeated the procedure every 8-12 hours for 7 days. A progressive reduction in the volume and purulence of the secretion was recorded. For 3 days Gentamicin alone was injected into the fistula, followed by the complete deflation of the implant cylinders. The opening closed after 20 days. We maintained the

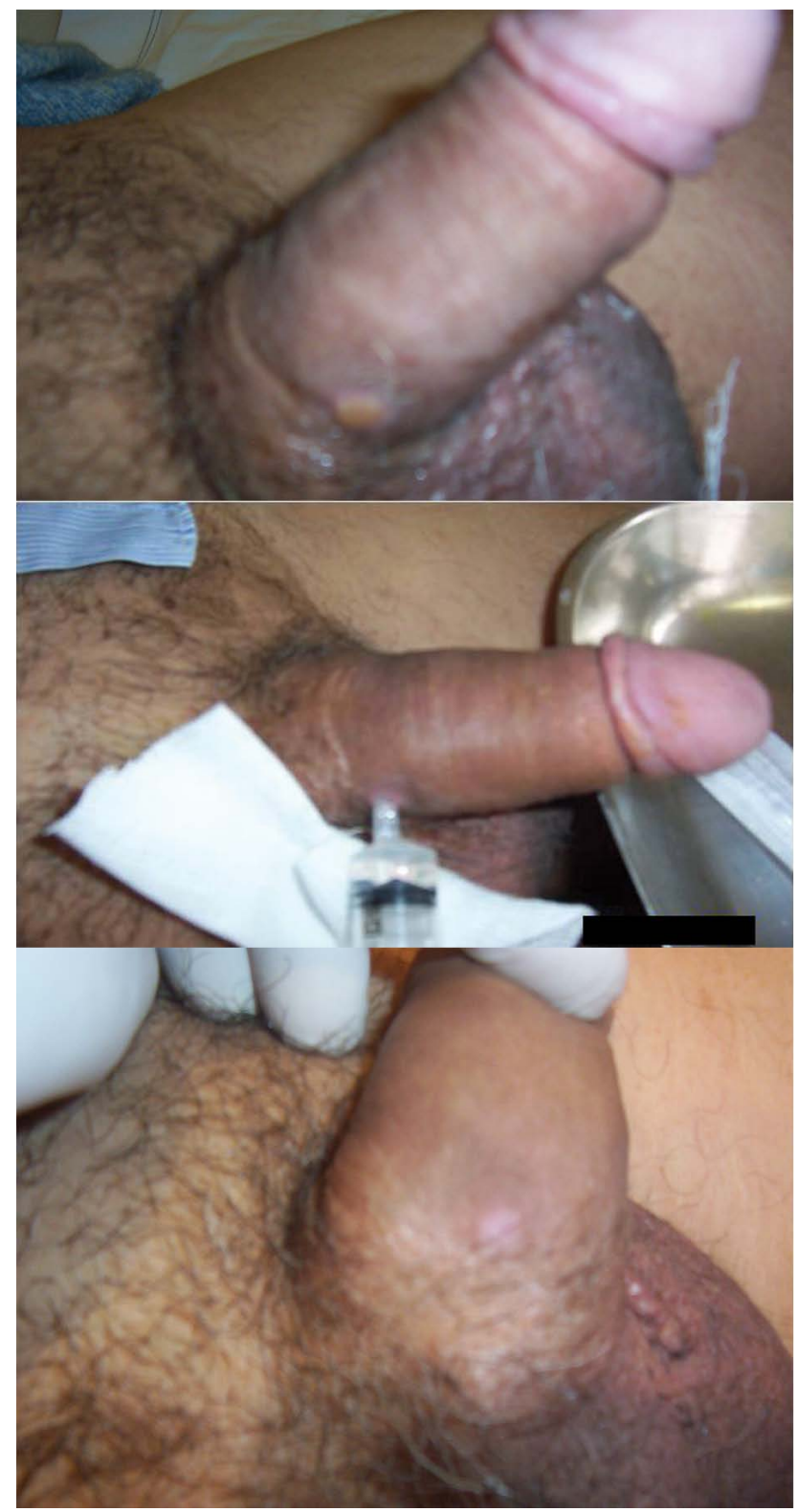

Figure 1: Cutaneous fistula with purulent secretion 15 days after IPP; Local infusion of iodopovidone and Gentamicin solution; Closure of the fistula after 20 days of medical treatment. 
cylinders at maximum inflation. No secretion, pain or fever was observed for 4 days with the deflated cylinders. The patient did not complained further infections and maintained and utilized the implant regularly from 2004 to the last control in 2012.

-In 2004, a 72 years old patient, diabetic, due to complete ED received an AMS700 Ultrex with a penoscrotal incision. After 2 years, in 2006, the patient started reporting fever, scrotal pain and leakage of purulent material from the old penoscrotal scar. We predicted the removal, but we began to inject lodopovidone solution and Gentamicin into the scrotal wound twice a day for 7 days, in addition to a general therapy with Ciprofloxacin and Ceftriaxone. The leakage of purulent material stopped, and the wound healed (Figure 2). After 15 days, the patient begun using the pump and the prosthesis again. Since then, IPP works regularly.

\section{Intraoperative Complications}

Apical perforation during corporal dilation is always

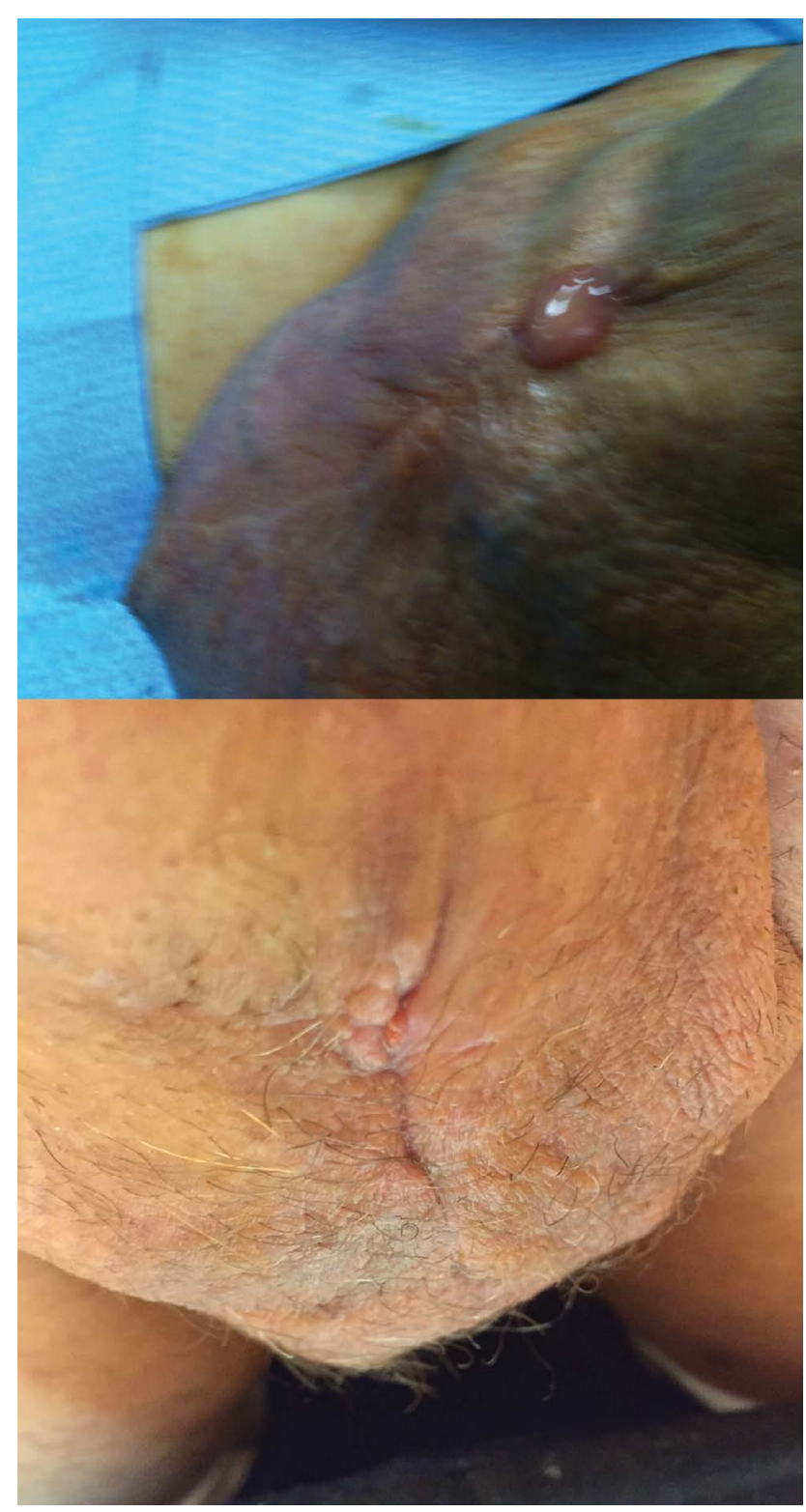

Figure 2: Purulent secretion from penoscrotal incision; After 7 days of local infusion of Gentamicin solution. possible. In our experience we did not registered such a complication.

Crural perforation was recorded in a 28 years old patient, affected by Corporal Veno-Occlusive Dysfunction (CVOD). Penoscrotal incision. During scissor and Hegar corporal dilation, the right crus of the penis was perforated. No perineal hematoma occurred. We inserted a Rear Tip Extender (RTE) in the crus and, with a small perineal incision, we affixed it to the tunica albuginea with Vicryl 3/0 stitches. We inserted Eurogest $11 \mathrm{~mm}$ cylinders: $21 \mathrm{~cm}$ on the left and $19 \mathrm{~cm}$ on the right. No postoperative complications were recorded. At 5-year follow-up the patient continued to be greatly satisfied.

\section{Septum perforation}

During implantations of malleable prostheses, in 36and 64-years old patients, we inserted the two rods in the same cavernous body due a false route accidentally performed, during Hegar cavernosal dilation, causing perforation of the cavernous septum. As soon as the complication was noted, we removed and re-inserted the prosthetic rod it in the correct cavernous space. No bleeding neither urethral complication occurred. Both patients did not refer any postoperative consequences.

\section{Immediate Post-Operative Complications}

\section{Postoperative hematoma or bleeding}

Hematoma formation in the post-operative setting is a potential complication for many surgical procedures, and it may contribute to post-operative complications following IPP placement. In patients under Aspirin or Coumadin therapy the incidence of bleeding is not insignificant, even though they are normally instructed to stop taking these drugs from 7 days before to 7 days after implantation. Hematoma or bleeding may be caused by: a) Scrotal vascular lesions occurring during access to the corpora cavernosa; b) Positioning of the prosthetic pump or reservoir; c) From the corpora cavernosa themselves through incompletely sutured corporotomy after semirigid or malleable penile prosthetic implant or through the necessarily incompletely closed corporotomy after IPP implantation. To avoid the risk of blood collections, short-term (24-36 hour), closed suction drainage has been proposed [11]. In our cohort of 402 semi-rigid, malleable or single-component Inflatable prostheses, we registered $3(0.7 \%)$ scrotal hematomas, both resolved without surgery.

1) After the malfunction of an AMS700CX, implanted at the age of 45 for vascular ED, a 52 years old patient underwent reimplantation of a similar IPP. The patient was on cardiovascular therapy. He was suggested to stop Aspirin 7 days previously. After penoscrotal incision, the cylinders and the pump were removed and replaced (maintaining the reservoir) without any peculiar problems. Suction drainage was used. Next 


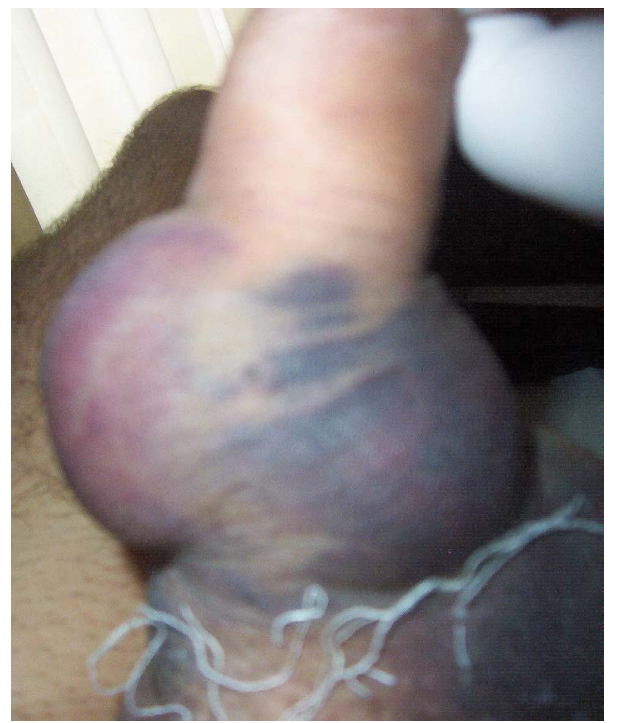

Figure 3: Basal Hematoma with diffuse ecchymosis: Medical therapy.

morning, 18 hours later, the drain had aspirated 30 $\mathrm{mL}$ of bloody fluid; no scrotal swelling. The cylinders, as usual, were deflated, and we preferred to maintain the drain till the afternoon, just before dismissing the patient who lived far away. Late in the morning a large scrotal hematoma $(150 \mathrm{ml})$ was recorded. The drain had aspirated $150 \mathrm{ml}$ of blood. The patient was transferred to the operating theatre; the bleeding was found to originate from the suture of the left corpus cavernosum. The prosthetic cylinder was fully inflated, and the bleeding completely stopped. The hematoma was drained, and the area flushed with antibiotics; a new suction drain was positioned. The next day there were no signs of hematoma or blood in the drainage. The patient was discharged and instructed to keep the cylinders inflated for 10 days and to suspend Aspirin for a further 15 days. Four years later the implant was still working well.

2) Coloplast malleable Genesis were implanted in a 31 years old man, without any relevant medical problem. A diffuse ecchymosis involving the pelvic and genital area, with localized hematoma, was observed the following day (Figure 3). We treated the vast ecchymosis with medical therapy and local creams. In few days it disappeared completely.

- A year later the same patient, dissatisfied with the rigidity of the Genesis implant, asked for a Coloplast OTR IPP. The removal of the Genesis cylinders occurred easily, as well as the insertion of the reservoir in the retropubic space and of the inter-testicular pump. Closed suction drainage was used. After few hours, a large hematoma appeared, involving the scrotal sac and the shaft; the drain was empty (Figure 4). An exploratory procedure was immediately planned but we failed to identify the source of the bleeding. After having flushed the area and having positioned a new suction drain the

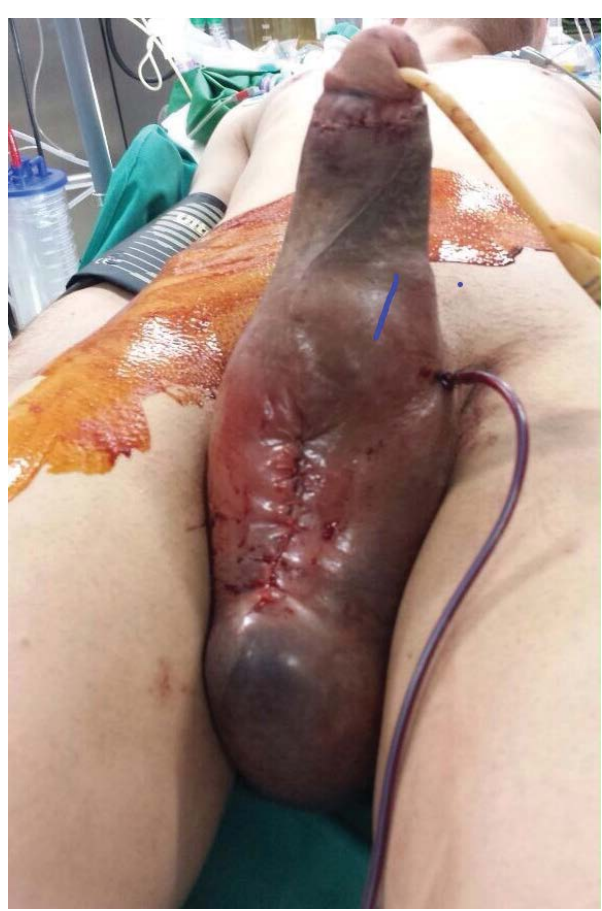

Figure 4: Massive Scrotal-penile Hematoma: Surgical Exploration.

bleeding stopped. We maintained he cylinders fully inflated. The suction drain, which remained empty, was removed after 2 days. The cylinders were deflated after 5 days, after which a complete recover of the hematoma was observed. At the last followup visit, two years later, the patient reported good penetrative activity.

3) Another case was observed in a 68 years old patient, who reported Acute Myocardial Infarction (AMI) six years earlier; good erectile response with PDE5is until he underwent Video-Laparoscopic-RadicalProstatectomy for Gleason $6(3+3)$ prostate cancer. After prostatectomy he didn't respond to PDE5i and reported a not satisfying response to PGE1 too. We programmed the implant of a Coloplast Titan OTR IPP. One week before surgery anticoagulant therapy was suspended. After penoscrotal incision, we found some difficulty in dilating the fibrotic retropubic space even with a Foley Catheter inflated to $100 \mathrm{ml}$. A $75 \mathrm{ml}$ reservoir was inserted without any reflux. The pump was positioned in the left scrotal space. The drainage was removed after 24 hours. After 7 days the patient recommenced anticoagulant therapy and he began to notice a painless scrotal swelling. The patient found it difficult to find the scrotal pump with his fingers. We checked the patient after 28 days. The scrotum was swollen $(18 \times 18 \mathrm{~cm})$ and painful. The pump was not localizable. US scan revealed an effusion with a corpuscular appearance surrounding the pump. We decided to re-operate. The left hemi scrotum was completely full of bloody fluid surrounded by a thick capsule. We cautiously resected the neo capsule and the area was flushed with hydrogen peroxide $\left(\mathrm{H}_{2} \mathrm{O}_{2}\right)$ and Gentamicin solution. The pump was positioned in a sub-dartoic right scrotal pocket. There 


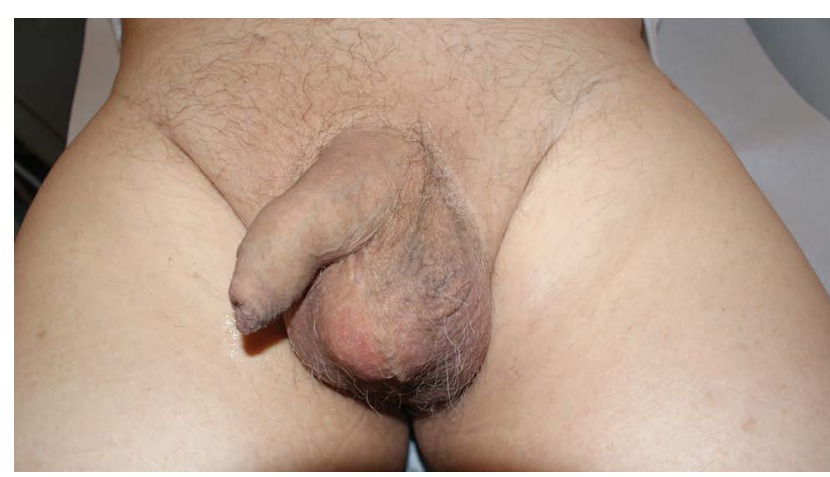

Figure 5: Hydrocele formation surrounding the scrotal pump: Surgery.

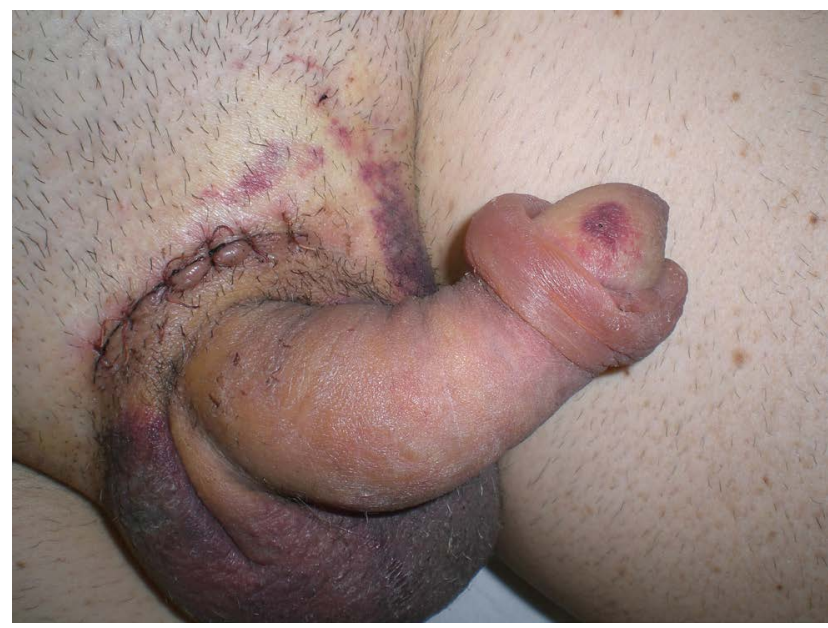

Figure 6: Foreskin lymphoedema 7 days after infra pubic incision: Initial medical treatment.

was no secretion from the wound and after 7 days the patient was able to palpate the scrotum and easily identify the testicles and the pump. After a month he was able to have satisfactory sexual intercourses.

4) Repositioning of the pump due to serous effusion. In 2008 we implanted an AMS700 Ultrex IPP on a 63 years old patient, with Peyronie's disease and shaft deviation. The patient was dismissed the next morning. No complications were recorded after 3 days. The patient performed numerous long car trips. 10 days after surgery, he complained because it was difficult for him to perceive the scrotal pump due to a progressively increasing of the scrotal volume. We checked the patient after 21 days (Figure 5). A US examination revealed an effusion of scrotal fluid, preventing the location and activation of the pump. We organized a surgical exploration. The prosthetic pump was found to be surrounded by a clear, transparent fluid with a tense capsule. The fluid was drained, and the capsule was removed; bleeding was controlled. The pump was working correctly and after aspiration and drainage the scrotum was sutured. The drain was removed the day after and the patient was discharged. The patient begun to utilize the pump and the IPP after 15 days.

Edema of the foreskin: During surgery, an infrapubic incision could determine a blockage of local lymphatic drainage. We implanted an AMS700 LGX through the infrapubic route on a 68 years old patient. After 7 days, the patient developed a significant edema of the foreskin (Figure 6), complaining a considerable local discomfort and difficulty in discovering the glans. Treatment with oral Cortisone for 10 days led to a moderate reduction in the swelling, enabling the glans to be uncovered. Ten days after the treatment, the edema had resolved fully. Another 53 years old patient, with Peyronie's who underwent implantation of an AMS700 LGX through infrapubic route, complained 5-days after surgery of prepuce swelling, that prevented its retraction from the glans, causing considerable discomfort. After treatment with oral Cortisone for 15 days the edema normalized. Another 52 years old, vascular patient underwent implantation of a Coloplast Titan OTR, through the infrapubic route. He developed a significant edema of the foreskin 8 days after surgery. After 10 days the lymphoedema was still present and Cortisone therapy were started. After 25 days the patient started sexual intercourses. Five days later the foreskin edema reappeared. Having checked that the edema persisted after medical therapy it was decided to perform circumcision, and this procedure resolved definitely the problem.

Moving reservoir: A 51 years old patient with vascular ED, received an AMS700 LGX IPP through the penoscrotal route. The reservoir was positioned, as usual, in the Retzius area. 8 days after surgery the patient felt an egg-shaped swelling in the right groin. This was found to be the reservoir, which had migrated there spontaneously. The implant worked perfectly, although when the cylinders were deflated a swelling in the right groin was observed, disappearing when the cylinders were inflated. During a new surgical procedure, the reservoir was emptied and removed through a small infra-pubic incision. It was then re-implanted in the retropubic space after opening the transverse fascia. To avoid its migration the reservoir was fixed to the transverse fascia. After 2 months the situation was normal, and the prosthetic system worked perfectly.

\section{Late Postoperative Complications}

Infrequent but serious postoperative complications following IPP insertion include pump, cylinders and reservoir complications, component failure/breakage, infection, malfunction, and bleeding.

\section{Apical extrusion}

A 56 years old patient, with Peyronie's disease and ED, underwent AMS700 CX implantation. Two cylinders $18 \mathrm{~cm}$ long with $+2.0 \mathrm{~cm}$ RTE were inserted. After 6 months the patient complained pain in the glans, which appeared deformed, due to suspected apical extrusion of the right cylinder (Figure 7). It was decided to perform exploratory surgery. This involved subcoronal in- 


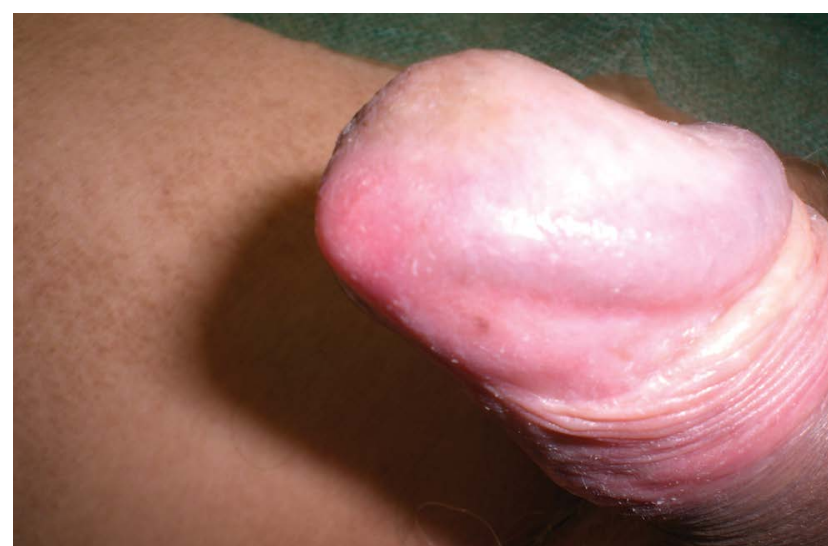

Figure 7: Partial extrusion of the Tip of AMS700: No infection.

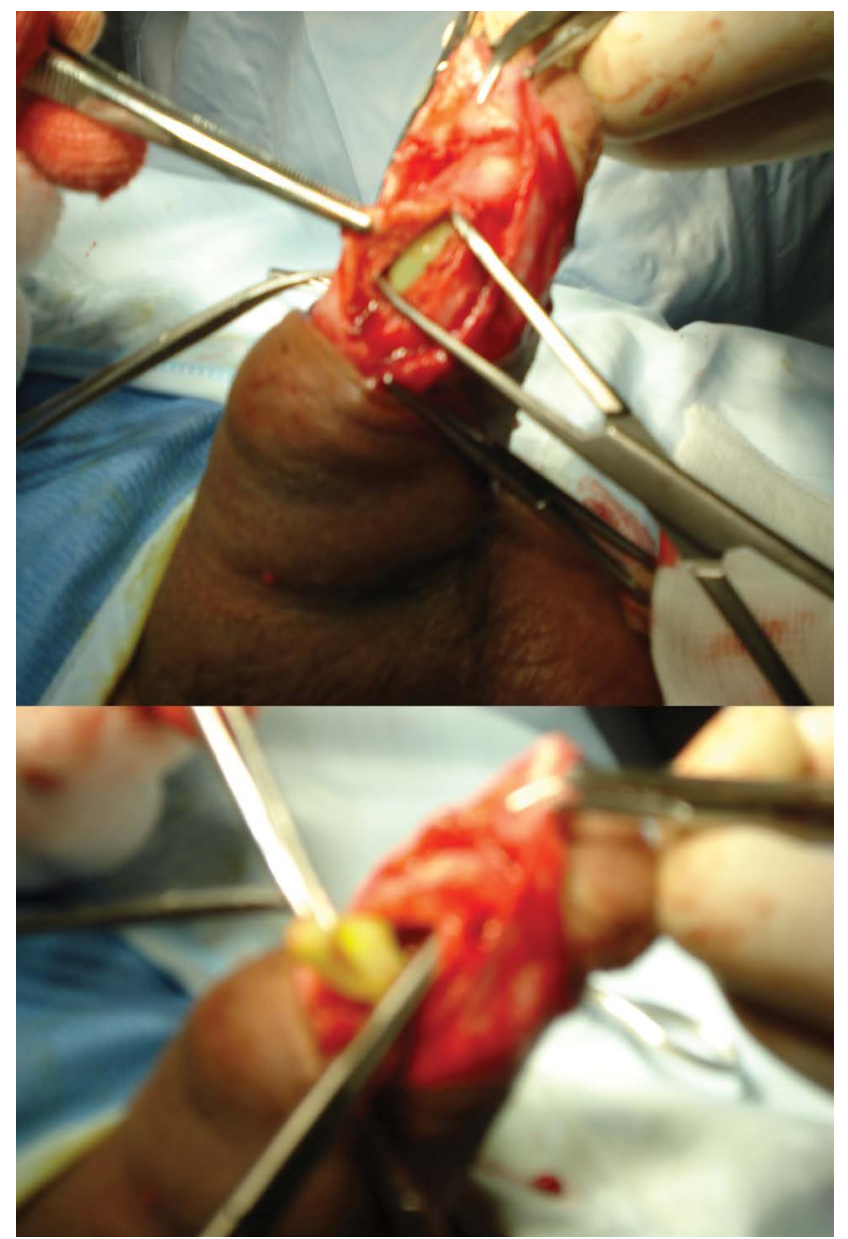

Figure 8: Distal incision to remove the cylinder.

cision, isolation of the glans to check the tip of the cavernous body which seemed to have been perforated by the cylinder. The cylinder was still inside the penis, and therefore there was no reason to suspect bacterial contamination. After incision of the right side of the penis and of the tunica albuginea, (Figure 8) the right cylinder was extracted and the $2.0 \mathrm{~cm}$ RTE was replaced with a $1.0 \mathrm{~cm}$ RTE. We affixed as a "hood" a small Marlex patch for hernia repair. The right cavernous cylinder was reinserted. After verifying that there was no apical tension, the glans was fixed above the tip of the corpus cavernosum. The cylinders were kept inflated for 5 days and

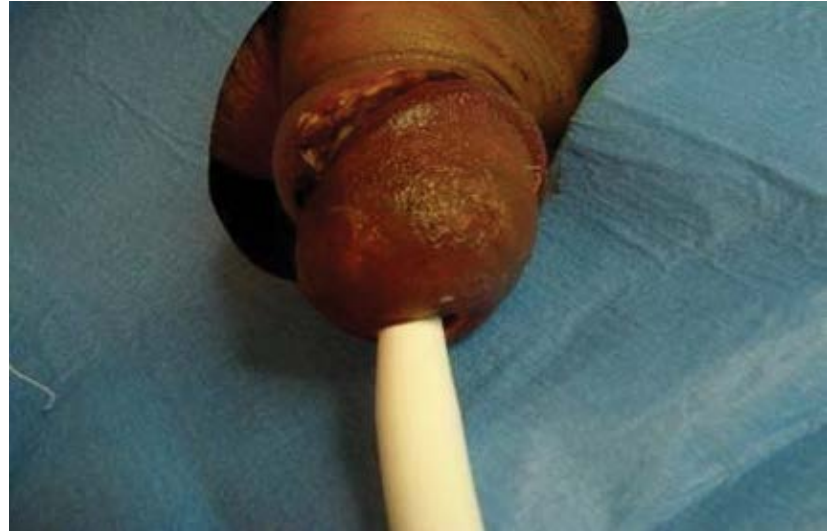

Figure 9: Extrusion of a malleable cylinder.

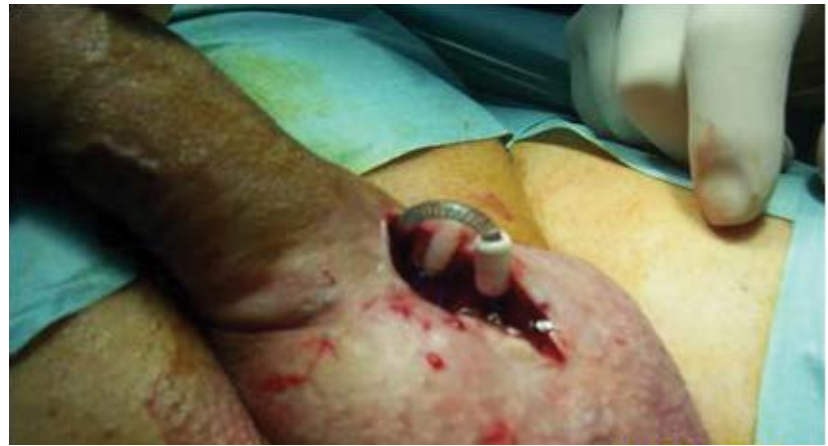

Figure 10: Scrotal skin perforation due to scrotal tubing: Local surgery.

then alternately inflated and deflated for several hours. After 30 days the patient was permitted to recommence penetrative sex. Seven years later, the patient was fully satisfied.

In a 36 years old patient with diabetes and CVOD, Implantal PP120 silicone rods were implanted in 2003. After 6 years he started complaining of pain in the glans, associated to the feeling of a lump on the left side of the penis. Surgery was performed to free the tip of the corpus cavernosum perforated by the cylinder, which was extracted, and the area was flushed with antibiotics. It was decided not to implant a new cylinder immediately due to the fear of infection. After discharge, the patient reported acceptable sexual relations with just one cylinder and preferred not to undergo further surgery (Figure 9).

\section{Tube erosion}

In a patient diabetic and hypertensive an AMS700 Ultrex IPP was implanted in 1999 with good sexual results till 2006 when he reported pain at the base of the penis with skin swelling and secretion of serous fluid. No fever. The Implant was correctly working. We perform a local surgical treatment. A small skin lozenge around the tube was removed (Figure 10). The skin was sutured over the buried tube. We didn't recorded any local complication and the patient, after one month, was allowed to utilize the IPP. 


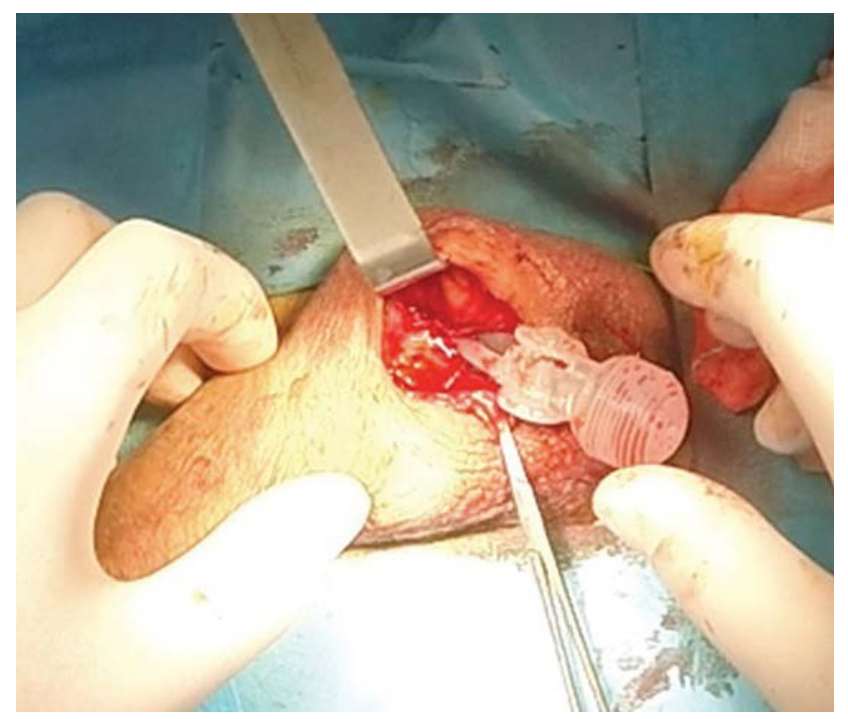

Figure 11: Repositioning of the pump.

\section{Adhesions of the tubing}

A patient, 72 years old, with CAD, Hypertension, Diabetes, Peyronie's disease with some curvature received a Titan-OTR IPP through an infrapubic incision. 10 days after the dismissal, at the check-up, a certain difficulty in finding the deflation button of the pump was reported. It was noted that a tract of the scrotal tubing settled on the deflation button, preventing an easy compression to deflate the prosthesis. We tried unsuccessfully to move the adhered little tube in various stages. It was decided to intervene, in Day Hospital, using local anesthesia with meticulous usual antibiotic therapy; median scrotal incision (Figure 11), freeing of the pump from adhesions, re-positioning of the little tubes. Skin suture. After 20 days it was possible to utilize the pump inflating and deflating the prostheses. After 1 year the patient referred satisfactory sexual intercourses.

\section{Urethral stones}

In 2017 the same patient who had a re-intervention for repositioning the scrotal pump in 2008, now aged 73 , under treatment for hypertension, hyperuricaemia and obstructive pulmonary disease, complained of an acute pelvic pain with an episode of gross haematuria. At the Ultrasound Sonography (US) of the abdomen in a different hospital performed by a MD not accustomed with Penile Surgery, kidney stones were excluded. It was evidenced a "liquid cyst" adjacent to the bladder and some hyperechoic images in the bladder. The day after the patient complained pain at the distal part of the penis with urinary retention. He returned to the author surgery. The IPP was perfectly working. The "liquid cyst" resulted to be the reservoir, inflating and deflating normally squeezing the scrotal pump. Two solid, roundish formations, obstructing the urethra were observed at US.

Using lidocaine cream, we performed a small

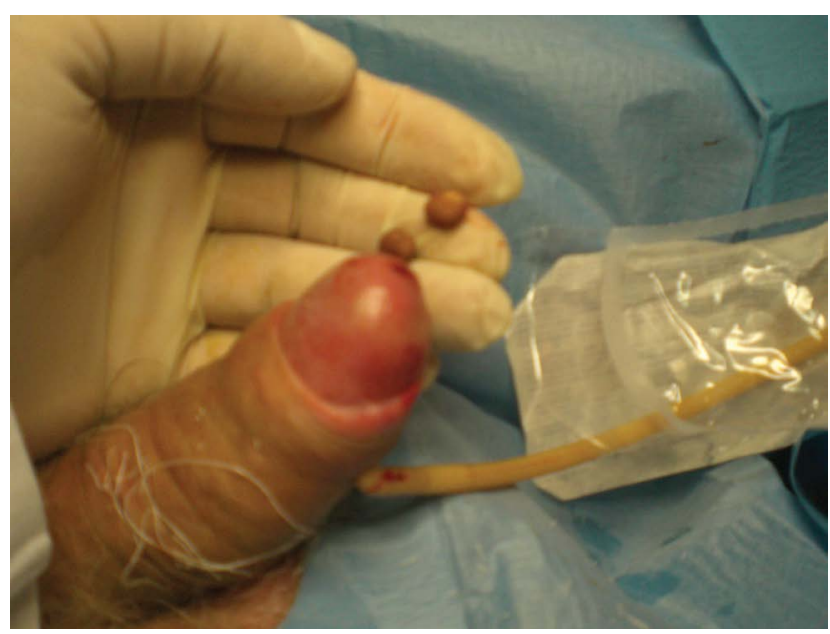

Figure 12: Two urethral stones removed 9 years after an IPP.

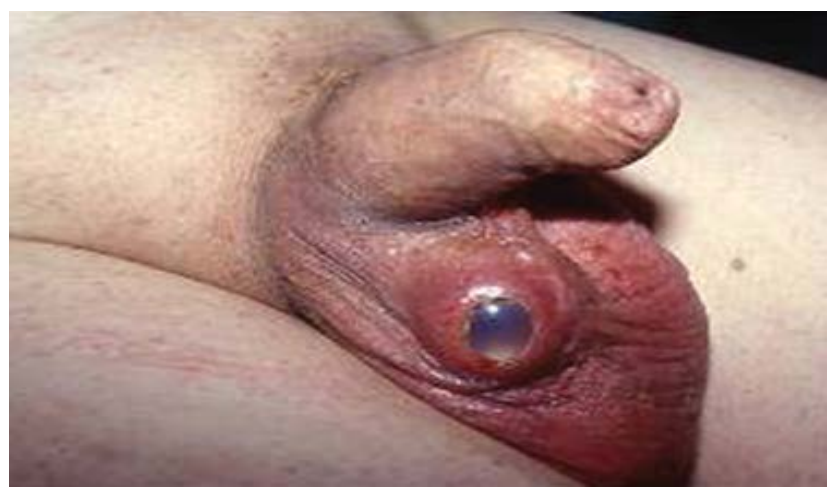

Figure 13: Purulent scrotal skin erosion due to scrotal abscess in diabetic patient 36 days after IPP: Removal of all the system.

meatotomy and two stones, of 5-7 mm diameter, were extracted (Figure 12). We inserted a Foley $\mathrm{CH} 16$ catheter without encountering any obstructions. Short antibiotic cycle of Ciprofloxacin for 5 days and the patient was submitted to flexible cystoscopy with no urethral strictures, prostatic hyperplasia, detrusor hypertrophy and no bladder stones.

\section{Scrotal skin erosion by pump}

In 2 diabetic patients, 58 and 64 years old, with AMS700 Ultrex IPP, after 30 and 36 days we recorded scrotal pain, inflammation, skin erosion and purulent reaction. In few days the prosthetic pumps emerged outside the scrotal skin (Figure 13). We decide to explant the IPPs. Neither patient wanted to undergo a new implantation procedure, due in part to financial motives.

\section{Trans Urethral Resection of the Prostate (TURP)}

3 of our patients (76, 78 and 82 years old) with IPP had to undergo to TURP for Prostatic Obstruction not-responding to the medical treatment. All the cases have been subjected to the usual antibiotic preop therapy with Ceftriaxone and Ciprofloxacin for 5 days. We did not observe complications in the prosthetic implants nor dif- 
ficult introduction of the Resectoscope. We never faced these kinds of problems with patients implanted with semi-rigid or malleable prostheses, where the introduction of resectoscopes could seem complicated of impossible for the urethral size and the penile fixated length.

\section{Psycho-emotional conditions}

We implanted a Coloplast Titan on a 53 years old Patient, MD, surgeon by profession: the patient din not have rigid erections due to CVOD not responsive at PDE5i. He had been in a relationship with a hospital colleague for 10 years without having any penetrative sex. He specifically requested an IPP. He had not informed his partner of his decision. A Coloplast Titan I was implanted through penoscrotal incision. He was dismissed the next day without any postoperative complications. After 1 month the IPP was working well. The patient could have his first penetrative intercourse.

After 2 months, the patient referred that his partner's reaction was completely negative. She was really upset and offended by his "prosthetic" decision. She told him that she had been fully satisfied with their long nonpenetrative sexual relation. She had threatened to leave him.

We suggested the patient to contact a psychologist, but he was fully determined and requested the explant. His reasoning was determined: "I'd rather have a woman without an implant than an implant and the risk of not finding another partner". After 5 years he contacted us in order to have a new prosthesis, but the difficulties linked with the cavernosal fibrosis made him to change the program.

\section{Common Complications}

Many malfunctions of the IPP caused the removal of the implant in this author's practice. Mechanical malfunction or breakage occurred in 14 of 18 (77.7\%) single-component (Hydroflex, Dynaflex) IPP and in 25 of 150 (16.6\%) multi-component IPP. Infections which could not be resolved by treatment were recorded in 15 of 168 cases of IPP (8.9\%). Six of 144 semi-rigid or malleable (4.1\%), 14 of 18 single-component IPP (77.7\%) and 23 of 150 multi-component IPP (15.3\%) were removed.

Surgeon's technical ability is fundamental, but an optimal surgeon-patient relationship is also important. In our experience, PPS does not end right after surgery. The patient should share with the surgeon all the aspects and problems observed before, while and after the operation. He should consult his surgeon also after the achievement of the correct sexual intercourse balance, which was fully recovered after prosthetic surgery, and for all the medical and surgical decisions, to maintain an ever-efficient penile prosthesis.

\section{Conclusions}

The main problem with PPS is represented by

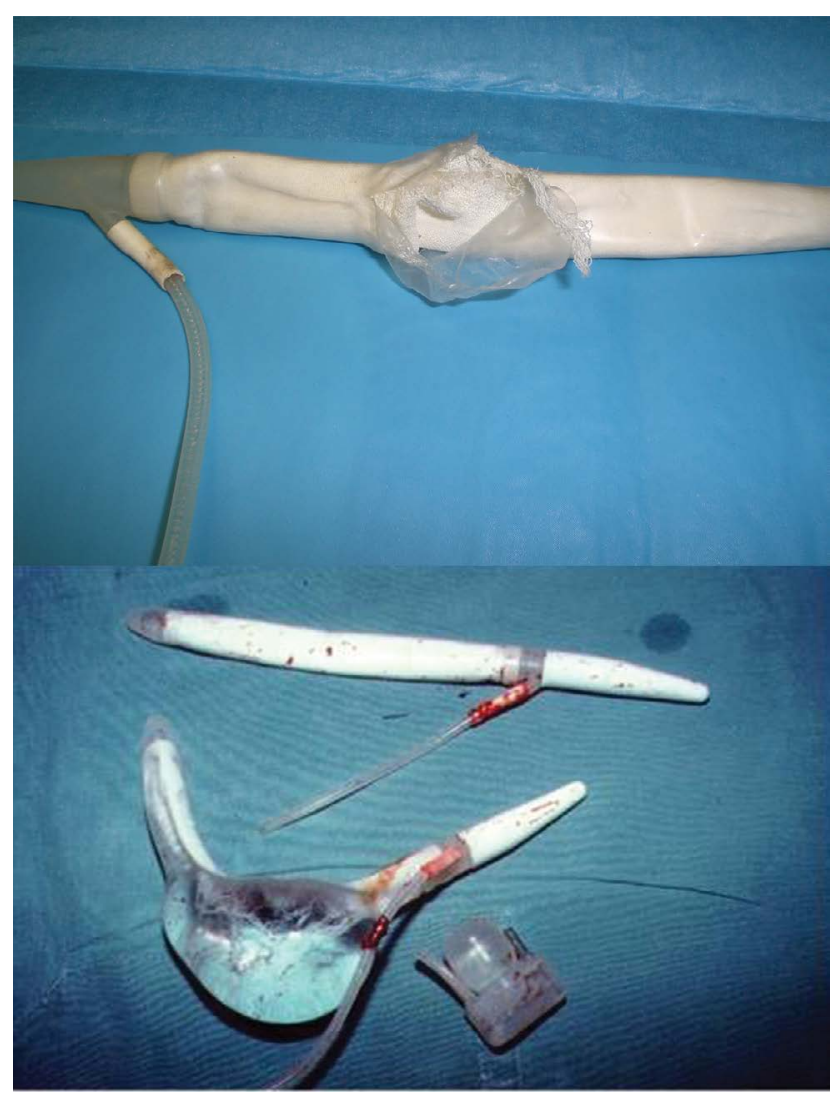

Figure 14: Rupture of the outer sleeve of AMS700 Ultrex after 5 Years; Aneurysmatic dilatation of AMS700 Ultrex cylinder after 3 years.

bacterial infections. Despite the scrupulous attention to antisepsis of the operating environment and of the patient, the use of thorough antibiotic prophylaxis, the new penile implants with antibiotic coatings, and the tendency to minimize operation times, the risk of infection has still not been eliminated, even with procedures performed by highly experienced surgeons [22].

The risks of malfunction, breakage or breakdown of the implant itself represent another limitation of PPS. After 15 years an IPP is likely to develop a problem (Figure 14). The lifetime guarantee offered by the manufacturers somewhat attenuates the annoyance of having to replace the implant but does not address the inconvenience and cost of the new procedure itself.

Some complications in PPS can be attributed to the surgeon's inexperience. While almost all surgeons will abide by the suggestions of their more expert colleagues when performing their first few procedures, their inexperience, indecision and need to repeat nonessential manoeuvres can lead to longer operation times and possibly excessive and negative surgical manoeuvres, movements and actions. In our experience some complications such as apical and crural extrusion, dislocation of the corpora cavernosa and perforations of the septum occurred during his early surgical career (Figure 15). After gaining more experience, patients with complex clinical situations who would in the past have 


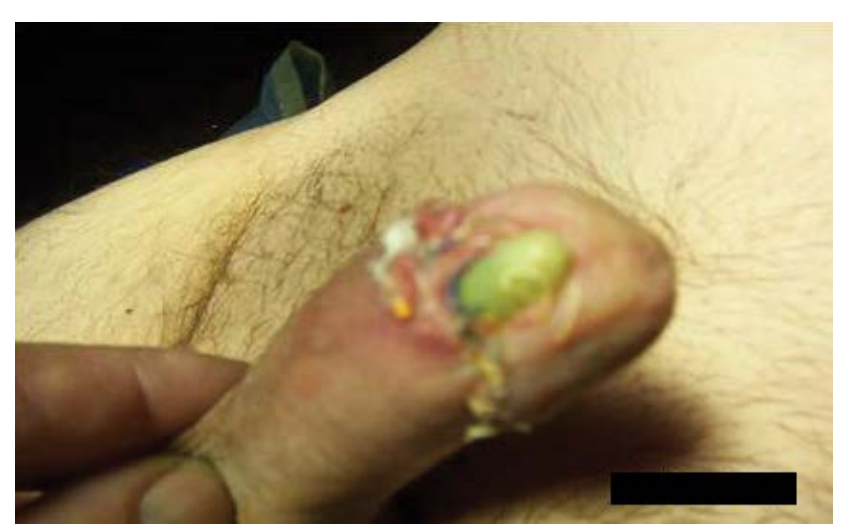

Figure 15: Apical extrusion with infection: Removal.

been sent to more comprehensive facilities, were also accepted. In the author's experience, not all infections must be considered an automatic cause for removal of the implant, as is often argued in the Literature [48]. In some selected cases, antibiotic therapies can be instigated to eliminate the responsible germs [5-7]. It is possible to eliminate a generalized sepsis and enabling the "sterilization" of the implant. Some complications could be related to tissue reactions involving the implant's components (extrusion of the cylinders or tubes, pump malfunction, involvement of scrotal pumps in reactive and coagulation processes) and could be resolved utilizing a limited surgical procedure.

\section{References}

1. Scott FB, Bradley WE, Timm GW (1973) Management of erectile impotence: Use of inflatable prostheses. Urology 2: $80-82$.

2. Small MP, Carrion HM, Gordon JA (1975) Small-Carrion penile prosthesis. New implant for management of impotence. Urology 5: 479-486.

3. Pearman RO (1972) Insertion of a silastic penile prosthesis for the treatment of organic sexual impotence. J Urol 107: 802-806.

4. Garber B (2005) Inflatable penile prostheses for the treatment of erectile dysfunction. Expert Rev Med Devices 2: $341-350$.

5. Ure I, Ozen A, Can C (2018) Life quality changes after inflatable penile prosthesis implantation. Aging Male 27: 1-7.

6. Mulcahy JJ, Kramer A, Brant WO, Parker JL, Perito PE, et al. (2014) Current management of penile implant infections, device reliability, and optimizing cosmetic outcome. Curr Urol Rep 15: 413-416.

7. Bodin T, Bruyere F (2015) Penile prosthesis systematic review of infectious complications. Prog Urol 25: 381-389.
8. Darouiche RO, Bella AJ, Boone TB, Brock G, Broderick GA, et al. (2013) North American consensus document on infection of penile prostheses. Urology 82: 937-942.

9. Dhabuwala C (2010) In vitro assessment of antimicrobial properties of rifampicin- coated Titan $(\circledR)$ Coloplast penile implants and comparison with Inhibizone(®). J Sex Med 7: 3516-3519.

10. Jani K, Smith C, Delk JR $2^{\text {nd }}$, Carson CC, Donatucci CF, et al. (2018) Infection retardant coatings impact on bacterial presence in penile prosthesis surgery: A multicenter study. Urology 119: 104-108.

11. Scherzer ND, Dick B, Gabrielson AT, Alzweri LM, Hellstrom WJG (2018) Penile prosthesis complications: Planning, prevention, and decision making. Sex Med Rev 19: 3005530056.

12. Narang GL, Figler BD, Coward RM (2017) Preoperative counseling and expectation management for inflatable penile prosthesis implantation. Transl Androl Urol 6: S869-S880.

13. Tran CN, Boncher N, Montague DK, Angermeier KW (2013) Erosion of inflatable penile prosthesis reservoir into neobladder. J Sex Med 10: 2343-2345.

14. Levine LA, Hoeh MP (2012) Review of penile prosthetic reservoir: Complications and presentation of a modified reservoir placement technique. J Sex Med 9: 2759-2769.

15. Hakky T, Lentz A, Sadeghi-Nejad H, KheraM (2015) The evolution of the inflatable penile prosthesis reservoir and surgical placement. J Sex Med 12: 464-467.

16. Stember DS, Garber BB, Perito PE (2014) Outcomes of abdominal wall reservoir placement in inflatable penile prosthesis implantation: A safe and efficacious alternative to the space of Retzius. J Sex Med 11: 605-612.

17. Ball TP Jr (1980) Surgical repair of penile "SST" deformity. Urology 15: 603-604.

18. Chung E, Van CT, Wilson I, Cartmill RA (2013) Penile prosthesis implantation for the treatment for male erectile dysfunction: Clinical outcomes and lessons learnt after 955 procedures. World J Urol 31: 591-595.

19. Pozza D, Pozza M, Musy M, Pozza C (2015) 500 penile prostheses implanted by a surgeon in Italy in the last 30 years. Arch Ital Urol Androl 87: 216-221.

20. Kramer A, Goldmark E, Greenfield J (2011) Is a closedsuction drain advantageous for penile implant surgery? The debate continues. J Sex Med 8: 601-606.

21. Khurgin J, Garber B (2014) Use of BioPatch ${ }^{\circledR}$ (Protective disk with Chlorhexidine Gluconate) in closed suction drainage for penile implant surgery. Curr Urol 7: 191-194.

22. Kashanian JA, Golan R, Sun T, Patel NA, Lipsky MJ, et al. (2018) Trends in penile prosthetics: Influence of patient demographics, surgeon volume, and hospital volume on type of penile prosthesis inserted in New York State. J Sex Med 15: 245-250. 